\title{
Lincoln à Cooper Union : Une analyse rhétorique du texte
}

Lincoln at Cooper Union: A Rhetorical Analysis of the Text

Michael Leff et Gerald P. Mohrmann

Traducteur : Sivan Cohen-Wiesenfeld

\section{(C) OpenEdition}

1 Journals

Édition électronique

URL : http://journals.openedition.org/aad/1078

DOI : 10.4000/aad.1078

ISSN : 1565-8961

Éditeur

Université de Tel-Aviv

Référence électronique

Michael Leff et Gerald P. Mohrmann, «Lincoln à Cooper Union : Une analyse rhétorique du texte», Argumentation et Analyse du Discours [En ligne], 6 | 2011, mis en ligne le 15 avril 2011, consulté le 23 septembre 2019. URL : http://journals.openedition.org/aad/1078 ; DOI : 10.4000/aad.1078

Ce document a été généré automatiquement le 23 septembre 2019.

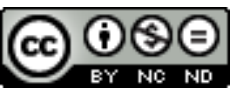

Argumentation \& analyse du discours est mis à disposition selon les termes de la licence Creative Commons Attribution - Pas d'Utilisation Commerciale - Pas de Modification 4.0 International. 


\title{
Lincoln à Cooper Union : Une analyse rhétorique du texte
}

\author{
Lincoln at Cooper Union: A Rhetorical Analysis of the Text
}

\author{
Michael Leff et Gerald P. Mohrmann
}

Traduction : Sivan Cohen-Wiesenfeld

\section{NOTE DE L'ÉDITEUR}

Cet article a paru en anglais dans Quarterly Journal of Speech 1974, $60: 3,346-358$, et est publié en français avec l'autorisation de la revue.

En guise d'introduction

Prononcé le 27 février 1860 dans la grande salle de l'Union Cooper pour le Développement de la Science et de l'Art ${ }^{1}$, peu avant la création du Parti républicain, les élections présidentielles de novembre 1860 et à la veille de la guerre de Sécession (1861-1865), le discours de Cooper Union prend place à une période riche en événements fondateurs de l'histoire des Etats-Unis. Aussi est-il nécessaire d'en rappeler le contexte politique, indispensable à la bonne compréhension de l'analyse proposée par Michael Leff.

Abraham Lincoln, alors avocat de campagne, originaire du Kentucky mais résident dans l'Illinois depuis l'âge de vingt ans, s'y opposera au sénateur démocrate Stephen A. Douglas, représentant de l'Illinois, sur le problème de l'esclavage. Cette question délicate avait fait l'objet de nombreux compromis législatifs fragiles entre le Sud esclavagiste et le Nord abolitionniste. À mesure que la jeune nation s'étendait vers l'ouest, les conditions dans lesquelles les nouveaux territoires seraient admis dans l'Union, en qualité de «territoire organisé », revêtaient une importance décisive. Dans l'histoire des États-Unis, un territoire organisé (organized territory) était un territoire pour lequel le Congrès américain avait voté un Acte organique, comprenant une déclaration des droits (Bill of Rights), qui en déterminait le système de gouvernement. Un territoire organisé différait d'un État américain par le fait qu'il possédait un gouvernement autonome limité, mais qu'il était soumis à la constitution américaine et que l'autorité ultime sur ce territoire était détenue par le Congrès. 
Dans le cadre d'un premier compromis, en 1821, le Missouri fut admis en tant qu'État esclavagiste, mais l'esclavage fut déclaré illégal dans toute la partie de l'ancienne Louisiane française située au nord de la frontière méridionale de cet Etat. Le "compromis de 1850 », quant à lui, comportait, en contrepartie de l'admission de la Californie comme État libre, l'adoption d'une nouvelle loi sur les esclaves fugitifs qui contraignait les tribunaux du Nord à faire procéder à leur arrestation et à les restituer à leurs propriétaires. En 1854, à l'instigation du sénateur démocrate de l'Illinois Stephen Douglas, la loi sur le Kansas et le Nebraska, annulant le Compromis du Missouri, remit aux habitants de ces territoires le soin de fixer leur statut d'États libres ou esclavagistes lors de leur entrée dans l'Union, en vertu du principe de la "souveraineté populaire".

C'est suite à l'adoption de cet « Acte du Nébraska » qu'eut lieu le meeting de Ripon (28 février 1854), qui donnera naissance quelques mois plus tard au nouveau Parti républicain, dont Lincoln fut l'un des dirigeants, formé après la dissolution du parti whig auquel il appartenait jusque-là.

Les affrontements entre les deux factions devinrent de plus en plus violents. Les tensions furent encore avivées par l'arrêt Dred Scott de la Cour suprême de mars 1857, déclarant que la Constitution ne conférait pas au Congrès autorité pour interdire l'esclavage dans les territoires. Esclave afro-américain que son maître avait emmené dans l'État libre du Wisconsin, puis ramené dans le Missouri, Dred Scott avait intenté une action en justice pour obtenir sa liberté, soutenant que son séjour dans le Wisconsin avait fait de lui un homme libre. Mais l'arrêt de la cour fixait qu'il n'était pas même habilité à intenter une action en justice devant un tribunal fédéral, n'étant pas citoyen des Etats-Unis, et ne possédant donc " aucun droit qu'un Blanc fût tenu de respecter ».

Cette décision suscita la fureur d'une grande partie des Nordistes, et Lincoln décida de se présenter à l'élection sénatoriale de 1858 contre le sénateur Douglas, favorable à l'arrêt de la Cour suprême. C'est alors qu'il prononça son célèbre discours « Une maison divisée ». Lincoln proposa à Douglas une série de sept débats publics dans différentes villes de l'Illinois, débats qui constitueront un épisode exemplaire de la démocratie américaine. Douglas l'emporte cependant par 54 voix contre 46 pour Lincoln. Ce dernier, peu disposé à battre en retraite, sillonne plusieurs États du Midwest tout au long de l'année 1859, attaquant la doctrine de la souveraineté populaire de Douglas et mettant en garde contre une future expansion de l'esclavage. C'est dans ce cadre qu'il se rend à New York, en février 1860, pour prendre la parole à la Cooper Union.

Il faut encore ajouter pour la compréhension du texte de M. Leff que l'investiture $\mathrm{du}$ Parti républicain pour la course à la présidence n'était pas gagnée pour Lincoln puisque le candidat présumé était le puissant William Seward, de New York.

Sivan Cohen-Wiesenfeld

1 Lorsqu'Abraham Lincoln prit la parole à la Cooper Union le soir du 27 février 1860, son auditoire réagit avec enthousiasme, et son discours ne cessa d'attirer les éloges au cours des ans. Biographes, historiens et spécialistes en littérature s'accordent pour affirmer que ce fut «l'un de ses discours les plus significatifs » (Randall $1945: 135)$, l'un de ceux qui illustrèrent «sa capacité de raisonnement " (Howard 1945 : 681), et celui auquel la postérité a attribué son "investiture et son élection ultérieures à la présidence » (Barondness $1954: 3)^{2}$. Ironiquement, cependant, ce modèle "d'analyse et de construction logiques» (Smith 1962:272) n'a pas suscité d'étude critique digne de lui. La plupart des études traitent du contexte, et trop souvent s'y mêle le mythe de l'homme public; pris dans le drame du spectacle, les auteurs n'ont épargné aucune information, s'attardant même sur des détails triviaux comme le prix des billets ou de 
la taille des nouvelles chaussures de Lincoln ${ }^{3}$. Si de tels détails peuvent nous aider à mieux apprécier l'événement, ils n'éclairent pas, cependant, le discours en tant que tel.

Lorsque l'on trouve des commentaires portant sur le texte lui-même, ils ne sont malheureusement pas plus éclairants. Nicolay et Hay affirment, par exemple, que les conclusions de Lincoln «étaient irrésistiblement convaincantes » (Nicolay \& Hay 1917 : 219-220), mais ils s'appuient sur un unique extrait composé de quatre cents mots. Et les analyses plus solidement étayées qui sont ancrées « fermement dans la tradition de la vénération du héros " (Hofstadter 1948: 364), ne valent pas mieux. Basler avance la curieuse hypothèse selon laquelle le " point culminant » rhétorique du texte se trouve situé vers la fin de sa première partie (Basler 1946:32); Nevins affirme de façon erronée que le discours "est divisé en deux moitiés " (Nevins 1950:186); des chercheurs réputés confondent le résumé et la citation avec l'explication (Randall 1945 : 136-137, Basler 1946: 32-33, Nevins 1950 : 186-187, Reinhardt 1960 : 210); et il est généralement admis que Lincoln a fait preuve d'une attitude conciliante envers le Sud (Randall 1945: 136, Barondness $1954: 18$, Nicholay \& Hay $1917: 220$, Nevins 1950 : 186, Luthin 1960 : 243-244).

3 Sans doute tout n'est-il pas à rejeter dans ces études, mais de quelque côté que l'on se tourne, on ne trouve dans la littérature critique aucune étude satisfaisante de ce discours ${ }^{4}$. Convaincus qu'une analyse rhétorique systématique peut remédier à la situation, nous offrons dans la suite de cet article notre propre tentative d'analyse. Si nous nous y concentrons sur le texte même du discours, notre objectif n'en appelle pas moins quelques remarques préliminaires sur son contexte rhétorique.

Bien que Lincoln n'ait franchement admis ses aspirations à la présidence qu'après ce discours - "J'en ai un peu le goût dans la bouche", dira-t- il ${ }^{5}$ - il en a savouré la possibilité pendant des mois. Au mois de novembre précédent, il écrivit que la prochaine campagne électorale le trouverait travaillant «fidèlement dans les rangs du parti » à moins que « la décision du parti ne m'assigne une position différente » ${ }^{6}$; mais alors même qu'il écrivait ces mots, il était avide de s'octroyer une autre tâche, "occupé à jouer du couteau contre ses rivaux... et faisant tout ce qui était en son pouvoir pour promouvoir sa réputation de brillant dirigeant républicain (Current $1958: 199)^{7}$. Il est dès lors peu surprenant qu'il ait rapidement décidé « d'en faire un discours politique " à New York ${ }^{8}$. Il avait là l'opportunité de se faire mieux accepter des Républicains de l'Est. Sans doute le fait de paraitre lui aurait-il suffit pour se faire mieux connaitre, mais la reconnaissance politique demandait plus; Lincoln devait être un Républicain acceptable, et offrir une alternative séduisante face au candidat des Démocrates.

5 Les candidats probables à l'investiture étaient William A. Seward et Stephen A. Douglas, et ils étaient les opposants déclarés de Lincoln. De plus, leurs opinions sur l'esclavage constituaient un danger qui menaçait à la fois sa conception du parti et ses ambitions personnelles. Les déclarations de Seward au sujet d'une "loi supérieure " et d'un "conflit irrépressible», heurtait le sens du conservatisme moral et politique de Lincoln; elles sentaient trop le radicalisme9. Douglas, de son côté, exacerbait la situation avec sa doctrine de la souveraineté populaire. Lincoln craignait que ce chant de sirène ne provoque une abjuration massive dans les rangs républicains, scénario somme toute assez probable si le candidat à l'investiture du parti paraissait trop radical. Il savait, cependant, qu'il existait une position intermédiaire, qu'il avait d'ailleurs longtemps tenue en affirmant que l'esclavage devait être protégé mais pas étendu. Par conséquent, lorsque Lincoln s'adressa aux Républicains de l'Est, les 
principes et l'intérêt du parti permettaient, voire dictaient, qu'il parlât aussi bien au nom du parti qu'en son nom propre, et qu'il maintint le parti et sa personne dans une position intermédiaire entre celles de Seward et de Douglas.

6 L'examen du texte du discours montre qu'il choisit cette direction, mais toutes les preuves extérieures montrent un homme travaillant de toutes ses forces, bien qu'avec humilité, en faveur de sa candidature politique, et lorsque Lincoln parla pour son parti, il parla d'abord pour sa propre investiture. L'allocution de Cooper Union est en fait, avant tout, un discours électoral: c'est un discours destiné à faire approuver la candidature de l'orateur. L'identification du genre de discours sélectionné est fondamentale pour notre analyse, et la nature du genre en question est suggérée par la distinction qu'a établie Rosenthal entre la persuasion non personnelle et la persuasion personnelle (1966 : 114-126) ; dans la première, l'orateur tente d'influencer l'attitude de l'auditoire sur un problème particulier, et l'ethos n'est important que dans la mesure où il ajoute foi à la substance de l'argument. Dans la seconde, le processus est inversé. L'accent est mis sur l'orateur, et le message devient un moyen de renforcer son ethos. Les discours électoraux, dans cette optique, sont plutôt des exemples de persuasion personnelle car, alors que «le but apparent d'un discours donné peut être d'obtenir l'adhésion à une politique particulière, [...] son but véritable est de gagner des voix pour le candidat» (Rosenthal 1966:120). En d'autres termes, le but final de l'orateur dans une campagne électorale est de faire sa propre promotion comme candidat. $\mathrm{Ce}$ sont à la fois la politique et la personne qui sont en jeu, mais la manière dont sont traités les problèmes est secondaire par rapport à l'objectif de créer une identification globale entre l'orateur et son auditoire. Le but d'un discours électoral est donc avant tout de s'insinuer dans les bonnes grâces du public.

7 En gardant à l'esprit le genre et l'objectif du discours, nous pouvons l'aborder à travers des notions familières. S'adressant d'abord au peuple de New York, puis aux sudistes et enfin au Parti républicain, Lincoln divise son discours en trois parties, et cette structure organisationnelle invite à une analyse successive des principales unités de disposition. De plus, l'argumentation et le style apparaissent immédiatement comme des éléments fondamentaux pour l'analyse, car ils révèlent des caractéristiques essentielles et des interrelations signifiantes entre les principales unités du discours. Par conséquent, notre étude critique suivra la structure organisationnelle proposée par Lincoln en mettant l'accent plus particulièrement sur l'argumentation et le style. Cette approche, cependant, n'est pas sans risques. Suivre l'ordre naturel de l'argumentation est aisé mais peut produire une analyse fragmentaire et brouiller le motif rhétorique dominant. C'est la prise en compte du genre qui permettra de pallier cet inconvénient. Son objectif essentiel est de recueillir les faveurs du public, et c'est ce but qui unifie les éléments de l'analyse en leur offrant un point d'ancrage plus précis ; c'est également la conscience de cet objectif qui éclaire la façon dont s'entrecroisent la structure, l'argumentation et le style.

8 Dans cette allocution, Lincoln traite exclusivement de l'esclavage, et bien qu'il puisse sembler que ce problème brûlant constitue une passerelle plutôt fragile pour s'insinuer dans les bonnes grâces du public, ce choix est en fait une réponse adaptée au problème rhétorique. Quel meilleur point de départ que cette suprême question, une question à l'ordre du jour avec laquelle sa personne était principalement identifiée, et qui avait engendré la création du Parti républicain ${ }^{10}$ ? Lincoln commence par le motif même qui avait attiré les gens à Ripon ${ }^{11}$ quelques années à peine auparavant, la question de 
l'esclavage dans les territoires qui entraient dans l'Union. S'appuyant sur ces associations de départ, il neutralise le caractère émotionnel de la question en adoptant une posture strictement rationnelle, et en énonçant un ensemble de principes modérés mais fermes. Cette approche le distingue de ses principaux adversaires et a pour but le renforcement de son alliance avec les Républicains de l'Est. Ce sont ces objectifs qui déterminent le choix et la forme de l'argument de départ, qui servira de base au développement ultérieur du discours. Dans le paragraphe d'introduction, et tout au long du texte, Lincoln s'associe, de même que les Républicains, aux pères fondateurs et aux principes constitutionnels, et en dissocie les factions et les candidats rivaux.

Remerciant ses « concitoyens de New York », Lincoln commence par adopter un « texte pour ce discours $»^{12}$. Il s'agit d'une déclaration de Stephen A. Douglas, dans laquelle celui-ci affirmait que "Nos pères, à qui l'on doit le régime sous lequel nous vivons, comprenaient la question tout aussi bien que nous, et même mieux» $(87)^{13}$. Redéfinissant les termes en séquence catéchistique, Lincoln affirme que la forme « du régime sous lequel nous vivons" consiste dans la Constitution et les «douze amendements qui furent adoptés par la suite » et que « nos pères » sont « les "trenteneuf" signataires du document original ». Il se demande alors quelle est cette question "que ces pères ont compris "tout aussi bien que nous, et même mieux" ? ». La réponse « est la suivante: la séparation bien comprise entre pouvoirs locaux et autorité fédérale, ou telle clause de la Constitution, interdit-elle au gouvernement fédéral de réguler l'esclavage dans les territoires fédéraux? » La question est liée au problème en cause, car «le Sénateur Douglas y répond par l'affirmative, et les Républicains par la négative ».

Il est plus exact de dire que Douglas devait en l'occurrence servir de repoussoir. La couverture de la campagne sénatoriale de 1858 par un journal national avait établi un rapport entre les deux hommes, et les débats devaient être publiés en mars ${ }^{14}$. Qui plus est, Lincoln avait poursuivi le débat tout au long de l'année 1859, s'inquiétant de savoir si le Parti républicain «maintiendrait [sic] son identité, ou se fracturerait pour devenir la queue du nouveau cerf-volant de Douglas $»^{15}$. Cependant, Lincoln savait que Douglas était vulnérable. La doctrine de Freeport avait convaincu de nombreux nordistes qu'il n'était que trop disposé à « subordonner les considérations morales à l'opportunisme politique» (Carman \& Syrett 1952: I, 588) ${ }^{16}$. Douglas était donc un rival attesté, que Lincoln percevait comme une menace pour l'unité du parti, et dont la position stratégique prêtait aux attaques au niveau de ses principes.

11 Au niveau tactique, le « texte » cité de Douglas fournissait à Lincoln un point de départ idéal. L'allusion aux pères fondateurs est une référence symbolique qui suscite un respect universel, et l'attaque implicite de Douglas contre les principes qui ont engendré le Parti républicain générait une antithèse au sein de laquelle orateur et auditoire étaient unis dans une opposition à un ennemi commun. Cette antithèse était un moyen pour l'orateur de s'insinuer dans les bonnes grâces du public ; Lincoln faisait du Républicanisme la voix de l'analyse rationnelle, et les termes précis de l'assertion de Douglas formaient les prémisses du questionnement logique. Poursuivant ce questionnement, Lincoln se lance dans une vigoureuse attaque ad hominem ${ }^{17}$. Il accepte la logique de Douglas, puis la retourne contre lui.

12 L'argumentation de la première partie est développée à partir d'une hypothèse unique : si la meilleure compréhension dont ont fait preuve les Pères fondateurs montre qu'ils pensaient que rien n'interdisait un contrôle fédéral de l'esclavage dans les territoires, 
cela signifie qu'un tel pouvoir régulateur est inhérent au système de gouvernement. Lincoln affirme cet antécédent par un raisonnement inductif élaboré. Les exemples qu'il donne consistent dans des actes des pères fondateurs avant et après la signature de la Constitution, car la question « ne semble pas avoir été portée directement devant la convention $»^{18}$. De l'Ordonnance du Nord-Ouest en 1784 au Compromis du Missouri de 1820, Lincoln énumère sept lois régulant l'esclavage dans les territoires, et rend compte des votes de vingt-trois des pères fondateurs ${ }^{19}$. Vingt-et-un d'entre eux votèrent en faveur d'une telle régulation. Etant donné que ces hommes étaient liés par " une responsabilité officielle et leur serments solennels » de fidélité à la Constitution, la conséquence à tirer de leur vote affirmatif ne fait aucun doute. Conclure que ces vingt-et-un pères fondateurs auraient approuvé une réglementation fédérale s'il l'avait trouvé inconstitutionnelle reviendrait à les accuser «d'une grave indécence politique et de parjure délibéré » et «si les actes parlent plus fort que les mots, de tels actes pris sous une telle responsabilité parlent plus fort encore ».

13 Mettant l'accent sur les actes et "adhérant rigidement au texte ", Lincoln ne peut apporter pour preuve « aucun jugement manifesté par qui que ce soit d'autre » que les trente-neuf signataires, ni citer les seize dont les votes n'ont pas été enregistrés. Mais ces derniers comprenaient des hommes comme Franklin, Hamilton et Morris, et il suppose que ce groupe « aurait probablement agi de la même manière que les vingttrois autres ». Quoi qu'il en soit, « une claire majorité d'entre eux » avait compris que rien «n'interdisait au gouvernement fédéral de réguler l'esclavage dans les territoires fédéraux ", et compte tenu du fait que les autres étaient probablement d'accord, il y a peu de doute sur «la conception de nos Pères fondateurs qui ont édifié notre Constitution d'origine; et le texte affirme qu'ils comprirent la question «tout aussi bien que nous, et même mieux ».

Lincoln utilise à présent cette compréhension pour discréditer les arguments fondés sur le cinquième et le dixième amendement à la Constitution; il affirme qu'il est " quelque peu présomptueux » de suggérer que les pères fondateurs auraient admis un principe en écrivant la Constitution et un autre en rédigeant ses amendements. Et estce que cette suggestion «ne devient pas impudemment absurde lorsqu'elle est associée avec l'autre affirmation, de la même source, selon laquelle ceux qui firent ces deux choses prétendument incompatibles comprenaient mieux que nous, mieux que celui qui affirme qu'elles sont incompatibles, dans quelle mesure elles l'étaient en effet ? » La touche de sarcasme révèle une attitude plus agressive, justifiée, cependant, par le processus inductif; le critère même sur lequel se fonde Douglas impose la conclusion qu'il n'a pas compris la conception des pères fondateurs. Lincoln deviendra même plus combatif encore avant la fin de la première partie, mais il faut à présent ajouter certains commentaires sur le style, qui nous conduiront à sa conclusion.

15 Le style de cette section est entièrement adapté à l'approche rationnelle très austère qu'adopte Lincoln. L'auditoire ne s'attendait probablement pas aux « feux d'artifice d'un discours électoral standard de l'ouest » (Nicolay \& Hay $1917: 2$, 220), mais Lincoln est particulièrement circonspect. Il ne propose aucune de ces "nombreuses excuses " qui en font un Uriah Heep ${ }^{20}$ aux yeux de certains de ses adversaires ${ }^{21}$ et évite toute ostentation, ne se permettant ni anecdotes, ni langage figuratif. La syntaxe est parfois complexe, mais cette complexité est celle de la prose légale plus que littéraire, comme on le voit dans la phrase suivante : «Par conséquent, il serait dangereux de considérer que même les deux personnes qui ont voté contre l'interdiction l'ont fait parce que, 
dans leur idée, une bonne répartition des pouvoirs entre autorités locales et fédérales, ou quelque clause dans la Constitution, interdisait au gouvernement fédéral de réguler l'esclavage dans les territoires fédéraux ».

Cette citation, qui fait écho au texte de Douglas, met en exergue un élément stylistique digne d'attention: la répétition. Lincoln utilise quinze citations in extenso sur la question et un nombre égal de citations tirées du «texte ", répétitions qui accentuent l'orientation univoque de l'argumentation. Il l'accentue davantage encore en insistant sur certains mots clés et sur certaines expressions. Il emploie, par exemple, plus de trente fois le radical "comprendre", généralement sous sa forme substantive "compréhension", et fait allusion aux "pères fondateurs" plus de trente-cinq fois. Aucune de ces répétitions n'est flagrante ni forcée parce qu'elles sont intégrées dans la texture du processus inductif. De plus, elles renforcent et contrôlent simultanément l'association émotionnelle avec les pères fondateurs et leur approche de la Constitution. Ce point est crucial pour l'évaluation de la pratique rhétorique de Lincoln. L'orientation de l'argumentation comme les symboles qui l'expriment sont puissamment émotionnels ; cependant, tout est intégré dans une structure logique et linguistique incisive et, bien que le ton reste rationnel et légaliste, il créé également un lien émotionnel subtil entre l'auditoire républicain et les pères fondateurs.

Comme nous l'avons noté plus haut, le style et l'argumentation changent dans les paragraphes de conclusion, une fois que Lincoln a bien établi ses références logiques. L'argumentation devient plus hardie, et le style se transforme parallèlement. Pendant le développement de sa réflexion inductive, Lincoln désigne les rédacteurs de la Constitution comme les " trente-neuf », mais ceux-ci redeviennent " nos pères » dans la conclusion du long premier paragraphe du discours. Les propositions deviennent alors plus soignées et plus sophistiquées :

Si aujourd'hui quelqu'un croit sincèrement [à l'inverse des pères fondateurs] qu'une séparation bien comprise entre pouvoirs locaux et autorité fédérale ou que telle clause de la Constitution interdisent au gouvernement fédéral de réguler l'esclavage dans les territoires, libre à lui de le dire et d'apporter, à l'appui de sa thèse, toutes les preuves crédibles et les arguments honnêtes qu'il est capable de rassembler. Mais il n'a pas le droit de tromper ceux qui ont moins que lui accès à l'histoire, et moins le loisir de l'étudier, en leur faisant croire que « les pères à qui l'on doit le régime sous lequel nous vivons" avaient la même opinion, en substituant ainsi le mensonge et la tromperie aux preuves véritables et aux arguments loyaux (87).

Ce passage achève la phase négative de l'argumentation de Lincoln. Le sujet aussi bien que la manière imposent sur le mode rationnel une division entre l'orateur et son adversaire. Lincoln suggère clairement que Douglas pourrait être coupable de " mensonge et tromperie », et il est tout aussi clair que sa propre position représente "des preuves véritables et des arguments loyaux ». Lincoln, qui faisait partie de ceux qui ont "accès à l'histoire " et "le loisir de l'étudier ", tente de mettre les choses au clair. Autre flèche décochée directe à Douglas: la source même du texte et du problème. En même temps, Lincoln se différencie sur le mode indirect de Seward et de sa posture radicale. La position de Lincoln est plus proche du droit et des exigences d'une recherche objective; elle tient également davantage compte des éléments à prendre en compte pour devenir un candidat plausible, et il est important de remarquer qu'il réalise cette dissociation sans avoir recours à une rhétorique de 
dissension. L'attaque contre la personne et sa position est patente, mais elle est de l'ordre de la pure inférence.

Bien qu'il n'ait pas le même degré de visibilité que la réfutation, un mouvement de construction important apparait dans cette partie du discours. Lincoln ne se distingue pas seulement de ses adversaires, il contribue également à renforcer l'unité républicaine en se présentant lui-même et son parti comme les canaux de transmission de la foi des pères fondateurs. Evitant de faire référence à sa propre personne, il se donne comme la voix du républicanisme, et il couronne son appel par des paroles qui sont tout à la fois adressées au parti, et issues de lui :

Mais cela suffit! Que tous ceux qui croient que nos pères, à qui l'on doit le régime sous lequel nous vivons, comprenaient la question aussi bien que nous, et même mieux, eh bien, que ceux-là parlent comme eux le faisaient et agissent dans cette affaire comme ils le firent. C'est tout ce que demandent les Républicains - tout ce qu'ils désirent - concernant l'esclavage. Comme l'ont fait nos pères, regardons à notre tour cette institution comme un mal qu'il faut se garder d'étendre, mais qu'il importe de tolérer et de protéger, ne serait-ce que, et dans la mesure où elle est effectivement présente parmi nous et que cette présence rend nécessaires ladite tolérance et ladite protection. Faisons en sorte que toutes les garanties données par nos ancêtres soient, non à contrecœur, mais pleinement et loyalement préservées. C'est pour cela que luttent les républicains et, si cela se produit, ils sauront, pour autant que je le sache et comme je le crois, s'en satisfaire (87-88).

A ce point du discours, Lincoln s'est associé, ainsi que son auditoire, avec l'esprit, les principes et les actes des pères fondateurs; il $a$, par là même, fait les premiers pas menant à la mise en valeur de sa personne.

21 Englobant près de la moitié du discours, ce paragraphe initial est d'une logique si claire qu'il est régulièrement cité comme un exemple du pouvoir de raisonnement de Lincoln. Mais ce serait sous-estimer grossièrement ses accomplissements que de s'en tenir là. Le paragraphe suivant est également remarquable pour son développement logique, et toute la suite du discours est anticipée et orientée par l'attaque contre Douglas. L'incapacité à distinguer cette unité a dérouté les commentateurs, dont la confusion est illustrée de manière flagrante par la conclusion généralement admise selon laquelle Lincoln aurait fait suivre son attaque de remarques "conciliantes envers le Sud» (Randall $1945:$ 136).

22 Le deuxième paragraphe commence bien par un ostensible changement de l'auditoire cible : «Et maintenant, s'ils veulent bien m'écouter, ce dont je doute, j'aimerais dire quelques mots à l'intention des gens du Sud ». Mais nous en apprenons davantage sur le véritable destinataire du discours lorsque nous nous apercevons que les vingt-six paragraphes suivants sont pleins de "termes d'avertissements et de protestations aimables " (Nicolay \& Hay $1917: 220$ ), de mots de « raisonnables et gentils pour dissiper les craintes du Sud » (Nevins 1950 : 186). Supposant qu'il ne serait pas entendu, Lincoln note que « notre parti n'obtient aucun vote » dans le Sud, et il affirme catégoriquement par la suite que «les sudistes ne veulent même pas nous entendre ». Ce ne sont pas là des réserves inutiles. Elles représentent au contraire l'évaluation réaliste d'un habile homme politique qui sait que le résultat des prochaines élections se décidera dans le Nord; et il est peu probable qu'un tel homme politique ait porté atteinte à son but suprême en adressant presque quarante pour cent de son discours à un auditoire hors d'atteinte. 
23 En réalité, l'auditoire n'a pas changé. Lincoln donne seulement à la seconde partie de son discours la forme d'une prosopopée, figure qu'il avait déjà testée cinq mois plus tôt à Cincinnati ${ }^{22}$. Ce dispositif convient admirablement bien à son propos. Il lui permet de simuler un débat entre les Républicains et le Sud, dans lequel il devient le porte-parole de son parti. Dans ce rôle, Lincoln peut renforcer son identification à l'auditoire républicain présent. Il prend soin de poursuivre la réfutation de Douglas dans cette seconde partie de son discours et reprend les lignes d'association et de dissociation qu'il avait auparavant mises en place. Il avait laissé peu de terrain à l'argumentation de Douglas et il use du même procédé envers le Sud; ainsi, le débat qu'il met en place est loin d'être conciliant.

La prosopopée prend la forme d'un nouvel argument ad hominem. Cette fois, cependant, la présentation en est compliquée par la nécessité, pour l'orateur, de faire face à une contestation issue d'une opposition collective. Pour en garder le contrôle, Lincoln commence de nouveau par mettre l'accent sur la raison, s'adressant au Sud: "Je considère que pour les vertus universelles de raison et de justice vous n'êtes pas inférieurs à n'importe quel autre peuple ». Cependant, dans ce cas précis, le discours rationnel est entravé par le fait que les sudistes ne font jamais référence aux Républicains si ce n'est pour «les dénoncer comme des reptiles, ou au mieux comme des hors-la-loi ». De telles réactions sont injustes pour les deux camps. La bonne attitude serait de "présenter vos accusations et vos demandes, et d'être suffisamment patients pour entendre nos dénégations ou nos justifications ». Clairement, le Sud est peu disposé et incapable de suivre cette procédure, et se donnant comme l'incarnation à la fois du Républicanisme et de la raison, Lincoln reconstruit les accusations et les charges particulières lancées contre eux, comprenant régionalisme, radicalisme, mise en avant de la question de l'esclavage et insurrections d'esclaves.

Le débat supposé commence: "Vous dites que nous avons un parti pris régionaliste. Nous le nions. Cela pose problème, mais la charge de la preuve vous incombe » (88). L'essentiel est de savoir si les Républicains repoussent le Sud à l'aide d' " un principe faux ». Mais le principe républicain est basé sur les croyances et les actions des pères fondateurs, et Lincoln défie le Sud de répondre à cela. «Acceptez-vous de relever le défi ? Non! Alors, vous croyez vraiment que le principe que "nos pères à nous devons le régime sous lequel nous vivons" tenaient pour si manifestement juste qu'ils l'adoptèrent avant de l'approuver encore et encore lors de leurs serments officiels, est en fait si manifestement faux qu'il requiert de votre part une condamnation aussi immédiate qu'inconsidérée?» (88). Clôturant et renforçant cette ligne de raisonnement, Lincoln fait référence au père fondateur prédominant: "Certains d'entre vous prennent plaisir à nous jeter au visage l'avertissement contre les partis régionalistes que Washington a formulé dans son discours d'adieu ", mais s'il pouvait parler lui-même, « attribuerait-il la responsabilité de cet esprit régionaliste à nous qui soutenons sa politique, ou à vous, qui la rejetez? Nous respectons, pour notre part, cette mise en garde de Washington, et nous vous la recommandons, ainsi que la façon exemplaire dont il veilla à ce qu'elle entre bien dans les faits » $(88)^{23}$. Le Sud prétend donc être la partie lésée, mais l'analyse de l'accusation prouve qu'il s'inflige lui-même ses blessures.

Lincoln utilise la même méthode de réfutation pour chacune des autres questions : définition de l'accusation par une série de questions rhétoriques, puis retournement de 
l'argument contre l'adversaire. Le Sud se proclame le bastion du conservatisme et dénonce le radicalisme républicain, mais

Qu'est-ce que le conservatisme? N'est-ce pas l'adhésion à ce qui est ancien et éprouvé, contre ce qui est nouveau et n'a pas fait ses preuves? Dans le domaine controversé, nous restons, nous, de fidèles défenseurs de la même vieille politique telle qu'elle fut adoptée par ceux de nos «pères à qui l'on doit le régime sous lequel nous vivons "; tandis que vous, vous repoussez comme un seul homme et rejetez sous les crachats cette ancienne politique tout en prétendant la remplacer par quelque chose de neuf (89).

Le Sud allègue que les Républicains ont aggravé le problème de l'esclavage. Il est vrai que le problème est aggravé, mais cette situation est apparue parce que le Sud «a abandonné l'ancienne politique des pères fondateurs ». Enfin, les sudistes prétendent que les Républicains fomentent la révolte parmi les esclaves, mais ils ne peuvent fournir aucune preuve à l'appui de cette allégation, pas plus qu'ils ne peuvent «impliquer un seul Républicain» ni ignorer que «les doctrines et les déclarations républicaines réfutent continuellement toute ingérence » dans les institutions des Etats esclavagistes. En fait, si ce n'étaient par les bruyantes et trompeuses protestations des politiciens sudistes, les esclaves auraient à peine connaissance de l'existence du Parti républicain. Pis encore, le Sud refuse de reconnaître une simple vérité contenue dans la doctrine républicaine, énoncée "il y a de nombreuses années " par Jefferson : la cause des insurrections d'esclaves est l'esclavage lui-même. Comme Jefferson, les Républicains ne souhaitent pas s'ingérer dans l'esclavage là où il existe, mais ils insistent, comme les pères fondateurs, sur le fait que le gouvernement fédéral «a le pouvoir de restreindre la prorogation de l'institution - et donc le pouvoir de garantir qu'une insurrection d'esclaves ne se produira jamais dans aucune des terres d'Amérique aujourd'hui exemptes d'esclavage » (92).

Après avoir traité des accusations spécifiques, Lincoln adopte un ton plus fort et plus agressif, semblable à celui qui caractérise la fin de la première partie. Ses réponses aux allégations sudistes sont elles-mêmes agencées de manière culminante, la question des insurrections étant à la fois la dernière et la plus critique. Toujours bouillant, ce sujet était devenu explosif depuis le raid de Harpers Ferry et le procès de John Brown ${ }^{24}$, et il est compréhensible que Lincoln en ait fait le levier principal de sa défense du parti et de ses principes. Cependant, il ne se contente pas d'adopter une simple posture défensive; l'analyse de l'ensemble du modèle de son argumentation révèle un mouvement qui mène de la réponse à l'attaque, et prend de plus en plus d'élan au fur et à mesure que le discours se déploie. Aussi, en ayant fini avec la controverse sur l'insurrection, Lincoln s'attaque au caractère même de la position sudiste, et conclut cette partie par un examen des menaces émanant du Sud.

Le Sud souhaite "briser l'organisation républicaine». Ceci ayant échoué, "vous entendez briser l'Union plutôt que de vous soumettre à une négation de vos droits constitutionnels " (92). Ceci relève bien sûr du principe "régner ou ruiner "; l'Union sera détruite si l'on n'autorise pas les Sudistes à amener des esclaves dans les territoires fédéraux. Mais un tel droit n'existe pas dans la Constitution, et les menaces $\mathrm{du}$ Sud sont infructueuses. Ni la Constitution ni le Parti républicain ne sont suffisamment flexibles pour se plier aux fantaisies du Sud. Même l'arrêt Dred Scott ${ }^{25}$ n'est ici d'aucune aide. Il s'agit d'un verdict rendu « par un tribunal divisé, à une faible majorité, et les juges n'étaient pas vraiment d'accord entre eux sur les raisons de son adoption ». La décision repose sur « l'opinion selon laquelle "le droit de propriété d'un 
esclave est distinctement et expressément affirmé par la Constitution" »; or une analyse attentive montre qu'un tel droit n'est même pas sous-entendu. Il est certainement raisonnable de s'attendre à ce que le tribunal revienne sur «cette déclaration erronée " lorsqu'il sera instruit de son erreur. De plus, le verdict est en contradiction avec le jugement des pères fondateurs, qui ont statué il y a longtemps sur la même question, "sans division entre eux lorsqu'ils prirent leur décision", sans division "sur sa signification après qu'elle fut prise", et sans "la fonder sur une présentation des faits erronée ». Ayant donc établi un contraste entre la tour de Babel du Tribunal et l'unité des pères fondateurs et de leurs descendants en ligne directe, Lincoln élabore une analogie frappante :

Dans ces circonstances, vous sentez-vous réellement autorisés à briser ce gouvernement à moins qu'une décision de justice comme celle que vous citez soit immédiatement adoptée comme ligne d'action politique irréfutable et définitive? Mais vous ne supporteriez pas l'élection d'un Président républicain! Si cela se produit, dites-vous, vous détruirez l'Union ; et vous ajoutez que la responsabilité de cet acte hautement criminel retombera sur nous. Un comble! C'est comme si un bandit de grand chemin me collait un pistolet sur l'oreille et me murmurait entre ses dents : "Debout et par ici la monnaie, sinon je te tue, et le meurtrier, ce sera toi !» (92)

30 Ajoutant que la menace du voleur de grands chemins ne peut " guère être distinguée en principe » de "la menace de destruction de l'Union ", Lincoln complète son attaque ad hominem contre la position du Sud, et la prosopopée arrive à son terme.

31 Les parallèles et les interrelations entre les première et deuxième parties du discours sont évidents. Le changement d'adversaire occasionne quelques modifications dans la sélection des moyens de persuasion et le style dans les deux parties, mais il est significatif que Lincoln choisisse dans les deux cas d'argumenter contre un opposant et qu'il utilise la même argumentation. Dans les deux parties, il s'efforce de devenir le porte-parole de son parti en démontrant qu'il est un homme de raison et que cette caractéristique l'identifie, lui et son parti, avec les principes des pères fondateurs. De plus, ce même trait le distingue des autres candidats. Enfin, chaque partie se fonde sur une structure rationnelle austère, et s'achemine vers un point culminant qui réunit les dimensions logique et émotionnelle en les intensifiant.

32 Mélangeant les styles et les types d'argumentation à l'intérieur du discours, Lincoln reste sans aucun doute en contact avec son auditoire direct, et ne perd jamais de vue la nécessité d'entrer dans ses bonnes grâces. Dans un premier temps, il se dissocie, ainsi que son parti, de Douglas et de Seward; dans un second temps, il fait contraster à son avantage la position de son parti avec celle de ses adversaires les plus fulminants ${ }^{26}$. Mais il restait encore une étape. Jusqu'à ce stade, l'orateur, le parti et ses principes ont été identifiés par une série de définitions négatives. Une détermination positive semblait nécessaire, et dans la dernière partie du discours, Lincoln unifie son auditoire à travers des appels plus constructifs.

Il commence par annoncer qu'il va maintenant «adresser quelques mots aux Républicains ", et bien qu'il laisse à présent de côté le texte de Douglas et le problème qu'il aborde, ses remarques découlent naturellement de ce qui précède. Une fois de plus, son point de départ est la raison. Après avoir, par sa métaphore du voleur des grands chemins, opposé raison posée et passion enflammée, il conjure les Républicains de "ne rien faire par passion et intempérance » qui pourraient causer la discorde au sein de la nation et, présentant les dernières implications des positions sudistes, il 
adopte l'antithèse comme mode d'argumentation et comme style. Toute la partie est centrée sur le contraste entre les Républicains et le Sud (entre " nous » et " eux »); elle développe et élargit la distinction entre les paroles et les actes présentée tout au long du discours; et l'argument est formulé et renforcé par une syntaxe antithétique.

Reconnaissant l'intransigeance du Sud, Lincoln n'en continue pas moins de demander à son parti de "considérer leurs exigences avec calme » et de parvenir à des conclusions basées sur tout «ce qu'ils disent et font». Poursuivant son examen, il demande: "Seraient- ils satisfaits si les Territoires capitulaient sans condition devant eux ? Nous savons qu'ils ne le seraient pas ». Et « Seraient-ils satisfaits si, à l'avenir, nous ne nous mêlions d'aucune invasion ni insurrection? Nous savons bien que non». Ils ne le seraient pas parce que notre abstention passée « ne nous a pas exemptés de ces griefs et de ces accusations ». Pour les satisfaire, "nous ne devons pas seulement les laisser tranquilles, mais aussi les convaincre d'une manière ou d'une autre que nous les laissons effectivement tranquilles ». L'expérience montre que ceci n'est pas une tâche aisée car la politique et les actions des Républicains ont été systématiquement mal interprétées. Le seul recours semble être "par ceci et par rien d'autre: qu'on cesse d'assimiler l'esclavage à un mal et qu'on se joigne à eux pour proclamer que c'est une bonne chose. Et pas question de faire cela à moitié : les actes devront suivre les paroles. Aucun silence ne sera toléré, et c'est ouvertement que nous devons nous ranger à leurs côtés» (93). Les Républicains doivent s'abstenir de tout "propos condamnant l'esclavage ", renvoyer "leurs esclaves fugitifs avec un plaisir de rapace ", et en finir avec les constitutions des Etats libres « avant qu'ils consentent à ne plus croire que tous leurs ennuis viennent de nous » (93).

La plupart des sudistes, admet Lincoln, ne présenteraient pas leur argumentation sous cette forme extrême. Ils se contenteraient de déclarer qu'ils veulent simplement qu'on les laisse en paix, mais "nous les laissons effectivement en paix ». Il apparaît donc clairement « qu'ils continueront à nous accuser d'actes [que nous n'avons pas commis], jusqu'à ce que nous nous taisions». Etant donné la nature de leurs arguments et le caractère de leurs actes, les sudistes ne peuvent pas ne pas aller jusqu'à exiger que tous les Républicains renoncent à parler et à agir selon leurs convictions. Ceux qui prétendent que « l'esclavage est moralement juste et constitue un facteur d'élévation sociale» (93) doivent nécessairement appeler à sa reconnaissance "comme droit juridique et comme bienfait social » (93). Dépouillé de son vernis et examiné à la froide lumière de la raison, la position du Sud révèle la divergence des vues qui préside à l'ensemble du conflit ; elle souligne également le principe sur lequel les Républicains ne peuvent céder. Lincoln exprime ces deux points dans une antithèse finale qui réduit le sujet de l'esclavage au problème du bien et du mal, à une question de conviction morale :

Toute la controverse repose précisément sur le fait qu'il [le Sud] [...] considère [l'esclavage] comme juste et que nous y voyons un mal. Si on pense, comme eux, qu'il est juste, il est normal de désirer qu'on le reconnaisse pleinement comme une institution légitime ; mais si on pense, comme nous, que c'est un mal, pouvons-nous leur céder? Pouvons-nous donner nos votes pour soutenir leurs vues et contre les nôtres? Au regard de nos responsabilités morales, sociales et politiques, pouvonsnous agir ainsi? identification et à celle de son parti avec les pères fondateurs, prenant Washington comme exemple. 
Le style change lorsque Lincoln en arrive à son appel final à l'unité. Des éléments antithétiques apparaissent dans l'avant-dernier paragraphe, mais les oppositions sont immergées dans le flot de la longue phrase finale composée de périodes, un flot qui réalise une union émotionnelle avec les paroles et les actes de Washington. Lincoln répète que l'esclavage peut être toléré là où il existe déjà, mais il insiste sur le fait qu'on ne peut atermoyer sur son extension :

Si notre sens du devoir nous l'interdit, alors n'écoutons que notre devoir, sans crainte et sans tiédeur. Ne nous laissons pas détourner par ces artifices pressants et sophistiqués qui nous assaillent - comme la recherche d'un compromis entre le vrai et le faux, aussi vaine que celle d'un homme qui ne serait ni vivant ni mort; comme la politique du "que m'importe" sur une question qui importe à tout homme véritable ; tels que les appels au nom de l'Union implorant les vrais Unionistes de se rendre aux partisans de la désunion, et inversant la loi divine en appelant non pas les pécheurs, mais les justes au repentir ; tels que des invocations à Washington, conjurant les hommes de taire ce qu'il a dit et de ne pas faire ce qu'il a fait.

$\mathrm{Ne}$ nous laissons pas détourner de notre devoir par les diffamations et les accusations mensongères portées contre nous ; ne cédons pas à la peur face à ceux qui menacent de détruire notre système politique et de nous jeter nous-mêmes au fond d'un cachot. Ayons foi dans l'idée que le droit fait la force et, soutenus par cette croyance, osons jusqu'au bout accomplir notre devoir tel que nous le comprenons (94).

Cette courte troisième partie, qui constitue moins de quinze pour cent de l'ensemble du texte, constitue un point culminant approprié aux efforts de Lincoln. Le principe rationnel est développée en conviction morale, et l'intensité émotionnelle qui en résulte émerge de tout ce qui précède et le synthétise. Pourtant, cette intensité émotionnelle reste contrôlée. L'orateur et l'auditoire sont résolus et fermes dans leurs principes, mais en même temps posés et logiques. Les autres peuvent se livrer à « de fausses accusations » et des "menaces de destruction", Lincoln et les Républicains garderont foi dans le droit et dans leur propre entendement.

Par cette évocation finale d'un comportement antithétique, Lincoln revient sur tout ce qu'il a dit précédemment, parachevant ainsi sa tentative pour entrer dans les bonnes grâces de son auditoire. Douglas donne l'exemple pitoyable d'un homme qui prône un principe malavisé de façon maladroite, et la notion de conflit irrépressible défendue par Seward est en contradiction avec le véritable esprit du Parti républicain, dont les paroles et les actes découlent de ce que les fondateurs du gouvernement ont dit et fait. Aucun de ces adversaires n'est à la hauteur de la nouvelle image de soi construite par l'orateur pour son auditoire. Qui plus est, Lincoln a, par sa performance, démontré qu'il est l'homme qui représentera le mieux le parti et ses principes. Partant des principes et de la raison, il a écarté les oppositions, différencié les Républicains des Sudistes, et encouragé l'unité du parti dans la foi qui nous fera « oser jusqu'au bout accomplir notre devoir tel que nous le comprenons ».

Les termes mêmes des paragraphes finaux reflètent le caractère organique de la quête de l'unité menée par Lincoln. "Comprendre » fait écho au «texte»; Washington est une évocation synecdotique des pères fondateurs; et le langage antithétique rappelle les dissociations fondamentales. En examinant le discours, nous avons tenté d'expliquer cette cohérence interne en retraçant la séquence des arguments et des images tels qu'ils apparaissent dans le texte, et en respectant la logique interne du discours. Nous avons la satisfaction de voir que cette analyse a pu produire une lecture plus exacte que 
les précédentes, permettant de mieux expliquer les raisons pour lesquelles l'Adresse de Cooper Union fut l'un des discours les plus significatifs de Lincoln.

Notre interprétation est, bien sûr, en contradiction avec l'explication conventionnelle de son attitude à l'égard du Sud. Là où d'autres l'ont trouvé conciliant, nous soutenons que sa position sur l'esclavage a été calculée pour gagner l'investiture, et non pas pour conquérir un public inaccessible. Le fait qu'il ait fait «de nombreuses déclarations similaires, et ne les ait jamais reniées $\aleph^{27}$, a indubitablement contribué à sa crédibilité auprès des Républicains, et par là à son investiture, mais sa position met en définitive en relief le conflit idéologique entre le Nord et le Sud. Certains Sudistes trouvèrent une consolation dans les assurances de Lincoln que l'esclavage serait toléré là où il existait déjà, mais les extrémistes perçurent le futur candidat comme la personnification du républicanisme noir, voire comme la source de la doctrine du conflit irrépressible ${ }^{28}$. Cette dernière perception était fausse. La nôtre l'est également, si nous ignorons les réalités de la rhétorique politique, et quelle qu'ait pu être par ailleurs la portée de ce discours, son objectif fut certainement de faire face aux problèmes immédiats posés par la campagne électorale.

Cette étude met l'accent sur le fait que les alternatives mènent parfois à l'exclusion, et que la rhétorique peut alimenter l'exclusion. Une telle perspective peut être gênante pour ceux qui veulent voir en Lincoln le Grand Conciliateur, mais nous sommes persuadés qu'une lecture correcte de l'adresse de Cooper Union exige une franche reconnaissance des motifs rhétoriques immédiats. En dépit de la mythologie, Lincoln était un être humain, dans toute la gloire du terme, et ce n'est pas le desservir que de concevoir ce discours comme un témoignage de son habileté politique, comme une preuve " qu'il était un opérateur ingénieux et adroit de la machine politique » (Donald 1956 : 65). Ce n'est pas non plus diminuer sa valeur en tant que littérature et qu'exposé logique. L'art politique et l'art rhétorique sont fonction l'un de l'autre, et la reconnaissance de cette fusion ne peut qu'améliorer notre compréhension du discours de Cooper Union. Et, en considérant l'ensemble de ce discours, nous nous contenterons de terminer par cette évaluation légèrement modifiée empruntée à un autre contexte : "Ce discours est, pour le dire aussi crûment que possible, un chef d'œuvre immortel » ${ }^{29}$.

\section{BIBLIOGRAPHIE}

Barondness, Benjamin. 1954. Three Lincoln Masterpieces (Charleston : Educational Foundation of West Virginia)

Basler, Roy P. (ed.). 1946. Abraham Lincoln: His Speeches and Writings (Cleveland : World)

Basler, Roy P. (ed.). 1955. The Collected Works of Abraham Lincoln (New Brunswick, NJ : Rutgers

University Press)

Beard, Charles A. \& Mary R. Beard. 1973. The Rise of American Civilization (New York : Mc Millan)

Argumentation et Analyse du Discours, 6 | 2011 
Brigance, William M. (ed.) 1943 A History and Criticism of American Public Address (New York : McGraw-Hill)

Buchanan, James.1960 [1908-1911]. The Works, ed. John Bassett More (New York : Antiquarian Press)

Carman, Harry J. \& Harold C. Syrett. 1952. A History of the American People (New York : Knopf)

Current, Richard N. 1958. The Lincoln Nobody Knows (New York : McGraw-Hill)

Davis, Michael. 1971. The Image of Lincoln in the South (Knoxville : University of Tennessee)

Donald, David. 1956. Lincoln reconsidered (New York : Knopf)

Dumond, Dwight L. (ed.). 1964 [1931]. Southern Editorials on Secession (Gloucester, Mass. : P. Smith)

Fehrenbacher, Don E. 1962. «Lincoln and the Formation of the Republican Party », Prelude to Greatness (Stanford: Stanford University Press)

Freburg Berry, Mildred. 1943. «Abraham Lincoln: His Development in the Skills of the Platform », Brigance, William M., 828-858

Freeman, Andrew A. 1960. Abraham Lincoln Goes to New York (New York : Coward-McCann)

Hofstadter, Richard. 1948. The American Political Tradition (New York : Knopf)

Holloway, Emory \& Vernolian Schwartz (eds). 1932.Walt Whitman, I Sit and Look Out. Editorials from the Brooklyn Daily Times (New York : Columbia University Press)

Johnston, Henry W. Jr. 1952. «Philosophy of Argumentum ad hominem », Journal of Philosophy 49, 489-498

Johnston, William Dawson. 1904. History of the Library of Congress, 1800-1864 (Washington :

Government Printing Office)

Lincoln, Abraham. 1905. Collected Works, eds John G. Nicolay \& John Hay (New York : Tandy)

Lincoln, Abraham. 2009. Le pouvoir des mots. Lettres et discours, présentés et traduits par Bernard Vincent (Paris : L'Archipel)

Jarrell, Randall. 1953. Poetry and the Age (New York : Knopf)

Mumford Jones, Howard \& Ernest E. Leisy (eds). 1945. Major American Writers (New York : Harcourt, Brace \& Co.)

Nevins, Allan. 1950. The Emergence of Lincoln (New York : Scribner's Sons)

Nicolay, John G. \& John Hay. 1917. Abraham Lincoln: A History (New York :Century)

Randall, James G. 1945. Lincoln the President (New York : Dodd, Mead)

Reinhardt, H. Luthin. 1960. The Real Abraham Lincoln (Englewood Cliffs, NJ : Prentice-Hall)

Rosenthal, Paul I. 1966. " The Concept of Ethos and the Structure of Persuasion », Speech

Monographs 33, 114-126

Sandburg, Carl. 1926. The Prairie Year (New York : Harcourt, Brace \& Co.)

Schopenhauer, Arthur. 1962. "The Art of Controversy », The Will to Live: Selected Writings (New York : R. Taylor)

Smith, R. Franklin. 1962. «A night at Cooper Union » Central States Speech Journal 13: 270-275

Ziley, Earl W. 1927. Four speeches by Lincoln (Colombus : Ohio State University Press) 


\section{NOTES}

1. College privé (établissement d'éducation supérieure, selon l'acception américaine) de NewYork fondé l'année auparavant par l'industriel Peter Cooper, inventeur prolifique, brillant entrepreneur, et l'un des plus riches hommes d'affaires américains de l'époque.

2. Le discours de Cooper Union est disponible en anglais en ligne sur: http:// www.americanrhetoric.com/speeches/abrhamlincolncooperunionaddress.htm [consulté le 1.4.2011] (Note de la rédaction).

3. Le compte rendu le plus connu dans ce genre est l'ouvrage de Sandburg (1926, II : 200-216), mais le plus complet est celui de Freeman 1960.

4. Freeman s'y réfère brièvement (1960 : 84-88), et bien que l'étude de Barondness s'étende de la préparation du texte à la réaction de l'auditoire (3-30), l'observation concernant Hofstadter (1948) lui est également applicable. Ziley (1927 : 15-27) étudie l'adresse, mais il limite son analyse à la première partie du discours, comme il le fait également dans son article publié dans American Public Address, vol. 2 (1943). Dans le même volume, le discours sert à Freburg Berry de base à un commentaire sur l'élocution (1943: 828-858).

5. Lettre à Lyman Trumbull, 29 avril 1860, Basler 1955 (4:45).

6. Lettre à William A. Frazer, $1^{\mathrm{er}}$ novembre 1859 , Basler $1955(3: 491)$.

7. Pour les indications sur l'activité de Lincoln, voir Basler 1955 (3:384-521).

8. Lettre à James A. Briggs, Basler 1955 (3: 494).

9. Voir la lettre à Samon P. Chase, 9 juin 1859, Basler 1955 (3:384); la lettre à Nathan Sargent, 23 juin 1859 (3:387-88); la lettre à Richard M. Corwine, 8 avril $1860(4: 36)$.

10. En 1954, « les whigs du nord, persuadés que leur vieux parti était moribond, des Démocrates las de la domination des planteurs et des abolitionnistes, pressés d'exclure l'esclavage des territoires organisés commencèrent à se rassembler pour résister au progrès du pouvoir des planteurs » (Beard \& Beard 1937 : II, 22 ; Fehrenbacher 1962 : 19-47).

11. Lieu de naissance du Parti républicain, formé en 1854 par des opposants à l'Acte KansasNebraska qui venait d'être voté, et qui remettait à la souveraineté populaire le soin de fixer le statut des territoires entrant dans l'Union, notamment concernant l'esclavage [Note de la traductrice].

12. Nous suivrons le texte des Complete Works, éd. Nicolay \& Hay (1905, V : 293-328), sans inclure de notes car, mises à part certaines exceptions sans importance, les citations se suivent. Ce texte est plus proche de l'original dans sa typographie que celui édité et publié comme document électoral par Charles C. Nott et Cephas Brainerd. Ce dernier est paru dans les Collected Works, III, $522-550: 1860$, p. 1. Pour les variations substantielles existant encore, voir aussi le New York Times du 28 février, les textes sont en minuscules, et cette constante mérite un commentaire. Lincoln a refusé les modifications qui lui avaient été suggérées dans l'original (Sandburg, II, 210 et 215-216) : il corrigea le manuscrit du journal (Freeman : 92-93); des tracts furent disponibles le premier avril (Collected Works, IV, 38-39); et Lincoln a inflexiblement résisté aux changements éditoriaux proposés par Nott (Collected Works, IV, 58-59). Ceci prouve le soin avec lequel il construisit son discours, mais suggère également qu'il prévoyait au début un public plus nombreux. Les pratiques de publication ainsi que sa propre expérience lui faisaient penser qu'il atteindrait ainsi beaucoup de ceux qui ne l'auraient pas entendu parler.

13. Les citations en français proviennent d'Abraham Lincoln, 2009. Les passages non traduits dans les extraits reproduits par cet ouvrage sont traduits par nous et ne comportent pas de numérotation de page (Note de la traductrice).

14. L'intérêt général pour les débats est souligné par la note éditoriale favorable parue dans le Brooklyn Daily Times, le 26 août 1858, et l'éditorial écrit par un certain Walt Whitman (I sit and Look out, Holloway \& Schwartz eds (1932: 96). Pour les lettres faisant référence à la publication des débats, voir Collected Works, III : 341, 343, 372-374, 515 et 516. 
15. Lettre à Lyman Trumbull, 11 décembre 1858, Collected Works : III, 345.

16. Voir Fehrenbacher $1962: 121-142$.

17. Les logiciens définissent souvent l'ad hominem comme un argument non valide résultant d'une attaque contre l'argumentateur et non contre la qualité de l'argument. Dans cet article, néanmoins, nous utilisons ce terme comme le fait Schopenhauer, qui considère l'argument ad hominem et l'argument ad rem comme les deux modes de réfutation fondamentaux. Il les distingue ainsi : "Nous pouvons montrer soit que la proposition n'est pas en accord avec la nature des choses, c'est-à-dire avec la vérité absolue et objective [ad rem] soit qu'elle est incompatible avec d'autres déclarations ou affirmations de notre adversaire, c'est-à-dire avec la vérité telle qu'elle lui apparait [ad hominem]» - Arthur Schopenhauer 1962, "The Art of Controversy ", The Will to Live: Selected Writings (New York: Taylor), 341 (ma traduction). Voir H. W. Johnston, Jr 1952 (489-498).

18. Lincoln savait sans aucun doute que James Wilson, Patrick Henry et Edmund Randolph avaient discutés le sujet (voir Collected Works, III : 526-27, n. 9), mais il a raison d'affirmer que le thème n'a pas été porté « directement » devant la Convention.

19. Pour Washington, en tant que Président, sa signature tint lieu de vote, de l'Acte de 1789 qui appliquait l'Ordonnance de 1787.

20. Uriah Heep est un personnage de David Copperfield, roman de Charles Dickens publié en 1849 , caractérisé par son humilité mielleuse, son obséquiosité et son hypocrisie. (Note de la traductrice)

21. Voir Hofstadter (1948: 94)

22. CollectedWorks, III : 438-454. Prenant la parole à Cincinnati, le 17 septembre, 1859, Lincoln s'adressa durant une si longue partie de son discours " aux habitants du Kentucky » (440) qu'un des auditeurs se plaignit à haute voix: «Parlez aux habitants de l'ohio, et pas à ceux du Kentucky!»(445). Nevins fait une intéressante analyse de la prosopopée dans ce discours, et note que Lincoln « parlait ostensiblement aux habitants du Kentucky (Nevins 1950 : 56).

23. Les diverses interprétations de l'avertissement de Washington et leur persistance sont illustrées, dans les débats, au début de l'année 1850, sur l'achat du manuscrit du discours d'adieu par la Bibliothèque du Congrès. La plus grande partie de ce débat est reproduite dans Johnston 1904 (I : 326-340).

24. Harpers Ferry est un village du comté de Jefferson en Virginie occidentale, connue grâce au raid de l'abolitionniste John Brown sur l'arsenal fédéral qu'il abritait à cette époque (Note de la traductrice).

25. Voir, plus haut, «En guise d'introduction».

26. Dans ce second mouvement, il poursuit son attaque implicite de Seward, et tous les auteurs relèvent l'imitation parodique du «principe de souveraineté populaire » [jeu de mot en anglais qui imite l'accent du sud de Douglas : « gur-reat pur-rinciple gur=canne à sucre, reat=gosse (note de la trad.)]. Buchanan est également pris pour cible ici, car il s'est fait le champion de la souveraineté populaire dans son « Troisième message annuel » du 19 décembre 1859 (Buchanan 1960 : X, 342). Les efforts de Lincoln furent firent leurs effet sur un reporter du Evening Post de New York, qui écrivit : «l'orateur place le Parti républicain sur le terrain même des auteurs de notre constitution et pères de notre République », et que "dans cette grande controverse, les Républicains sont le véritable parti conservateur». Ce reportage fut republié dans le Chicago Tribune du $1^{\mathrm{er}}$ mars 1860 , p. I.

27. Abraham Lincoln, « First Inaugural Address », Collected Works IV : 263.

28. Michael Davis (1971 : 7-40) retrace les vues du Sud depuis l'investiture jusqu'à l'inauguration présidentielle. Voir Dwight L. Dumond (1964 : 103-105, 159-162 et passim).

29. La citation originale portait sur un commentaire de Jarrell Randall du poème "Provide, Provide » de Robert Frost (Randall 1953 : 41). 
INDEX

Keywords : audience, campaign oration, Lincoln (Abraham), rhetorical analysis, slavery Mots-clés : analyse rhétorique, auditoire, discours électoral, esclavage, Lincoln (Abraham) 